\title{
La medicina personalizzata nel trattamento dell'acromegalia
}

\author{
Salvatore Cannavò ${ }^{1}$
}

Pubblicato online: 3 aprile 2018

(c) Springer International Publishing AG, part of Springer Nature 2018

\section{Commento a:}

Personalized medicine in the treatment of acromegaly.

L. Kasuki, L.E. Wildemberg, M.R. Gadelha.

Eur J Endocrinol (2018) 178:R89-R100

Il trattamento dell'acromegalia è fondato su un approccio multidisciplinare che può prevedere l'impiego della chirurgia, della terapia medica e della radioterapia. La scelta di tali opzioni è codificata in algoritmi sufficientemente definiti nelle linee guida internazionali e prevede l'impiego della terapia chirurgica ipofisaria come approccio di prima linea in quasi tutti i pazienti, con l'eccezione di coloro che presentano una ridotta probabilità di cura, un eccessivo rischio anestesiologico o che rifiutano tale tipo di trattamento, seguito dalla terapia medica con analoghi della somatostatina di prima generazione (SSa), octreotide LAR o lanreotide autogel. Nei casi che dimostrano resistenza a questi farmaci, dopo un adeguato incremento del dosaggio e un periodo di trattamento sufficientemente prolungato, trovano indicazione farmaci di seconda linea, pasireotide LAR e/o pegvisomant, e radioterapia, oggi in genere stereotassica. Nei tumori in progressione e refrattari a tali trattamenti può essere necessario anche un ulteriore intervento chirurgico. In considerazione della relativamente bassa percentuale di successo dimostrata dalla chirurgia, soprattutto nei tumori ipofisari di maggiori dimensioni, o dal trattamento con SSa, in molti casi il raggiungimento di un adeguato controllo di malattia e, quindi, di una significativa riduzione del rischio di morbilità e mortalità nonché del miglioramento della qualità di vita, è notevolmente ritardato e in alcuni casi si ottiene dopo anni dalla diagnosi.

L'articolo di Kasuki et al. richiama la nostra attenzione sulla necessità di utilizzare nuovi concetti di medicina personalizzata e di precisione nella elaborazione degli algoritmi terapeutici per i pazienti acromegalici, in analogia a quanto oggi viene proposto per il trattamento di altre neoplasie. Gli autori sottolineano come tale opportunità sia offerta da un migliore utilizzo di fattori predittivi già noti, quali la densità di granulazione del tessuto tumorale $\mathrm{o} i$ livelli di GH ma, soprattutto, dalla possibile individuazione di nuovi biomarker di aggressività dei tumori ipofisari GH-secernenti, di tipo molecolare o epigenetico, e di resistenza/sensibilità ai farmaci, quali si stanno dimostrano per esempio alcuni microRNA (ma non solo) che possono essere misurati non solo nel tessuto tumorale ipofisario ma anche nel circolo. L'adozione di nuovi criteri di indirizzo delle scelte terapeutiche, di tipo istologico o basati su fattori predittivi ricavati mediante "biopsia liquida", potrebbe consentire infatti di raggiungere un adeguato controllo di malattia in tempi estremamente più brevi rispetto a quanto si verifica attualmente in molti casi, evitando terapie inefficaci e eventi avversi, e di ridurre ingiustificati costi diretti (farmaci inefficaci) e indiretti (gestione delle comorbidità e inabilità).
S. Cannavò

cannavos@unime.it

1 Dipartimento di Patologia Umana dell'Adulto e dell'Età Evolutiva "G. Barresi”, Università di Messina, Messina, Italia 